\title{
Efficacy of Amniotic Membrane Transplantation for the Treatment of Corneal Ulcers
}

\author{
Kaspar Schuerch, MD, Andrea Baeriswyl, Beatrice E. Frueh, MD, and Christoph Tappeiner, MD
}

Purpose: To evaluate the outcome of amniotic membrane transplantation (AMTX) as a treatment for corneal ulcers.

\begin{abstract}
Methods: Patients treated with AMTX for refractory corneal ulcers between 2012 and 2017 were evaluated in a retrospective analysis. Primary outcome measure was complete reepithelialization.
\end{abstract}

Results: A total of 149 patients were included (mean age $68 \pm 18$ years). The mean duration between ulcer onset and AMTX was $42 \pm$ 46 days. The longest time between ulcer diagnosis and AMTX was found in bacterial ulcers and the shortest time to AMTX in eyes with trauma/chemical burns (mean $65 \pm 15$ days and $14 \pm 4$ days, respectively). In $70 \%$ of the patients, a single AMTX procedure was sufficient to achieve epithelial closure $(21 \%<1$ month, $40 \%$ within $1-3$ months, and 9\% within 3-6 months). Treatment failure was observed in $30 \%$ of all patients, and most of them underwent further interventions. Highest closure rates were found in bacterial ulcers, herpetic ulcers, and neurotrophic ulcers $(80 \%, 85 \%$, and $93 \%$, respectively), whereas the lowest reepithelialization rates were found in ulcers after corneal surgery and ulcers associated with rheumatic disease (52\% and $57 \%$, respectively).

Conclusions: AMTX is a valuable treatment option to achieve corneal epithelial wound healing in cases refractory to conventional treatment. Success rates differ depending on the etiology of ulcer.

Key Words: amniotic membrane, corneal ulcer, corneal disease, cornea, amniotic membrane transplantation, ocular surface disease

(Cornea 2019;00:1-5)

$\mathrm{T}_{\mathrm{p}}^{\mathrm{h}}$ he initial event leading to a corneal epithelial defect that progresses to ulceration is the breakdown of the corneal epithelial surface.1,2 A corneal epithelial defect may be caused by exogenous factors such as infection, ocular xerosis, trauma, or chemical burn. On the other hand, it may be because of endogenous conditions such as exposure to keratitis or neurotrophic keratitis, keratomalacia, or recurrent corneal erosions. ${ }^{3}$ When associated with excessive inflam-

Received for publication July 10, 2019; revision received September 3, 2019; accepted September 5, 2019.

From the Department of Ophthalmology, Inselspital, Bern University Hospital, University of Bern, Switzerland.

The authors have no funding or conflicts of interest to disclose.

Correspondence: Kaspar Schuerch, MD, Department of Ophthalmology, Inselspital, Bern University Hospital, University of Bern, 3010 Bern, Switzerland (e-mail: kasparschuerch@insel.ch).

Copyright (C) 2019 Wolters Kluwer Health, Inc. All rights reserved. mation or failure of the normal corneal healing response, the epithelial breakdown may eventually progress into a persistent corneal ulcer. ${ }^{4}$ Because of further corneal melting, such ulcers may end in a descemetocele or even in corneal perforation. All of these situations bear a high risk of potentially irreversible visual loss. ${ }^{1}$

Because etiologies of corneal ulcers may vary, early diagnosis and appropriate medical treatment are crucial. ${ }^{3}$ To further support epithelial healing in cases refractory to local medical therapy, the use of bandage contact lenses, ${ }^{5}$ surgical approaches such as conjunctival flaps ${ }^{6}$ or tarsorraphy, ${ }^{7}$ and eventually systemic immunosuppressive/-modulatory treatment $^{8}$ are established treatment options. Transplantation of amniotic membranes (AMTX) has been shown to be a promising surgical procedure that is helpful to reconstruct the ocular surface by supporting the epithelialization of the cornea. ${ }^{4,9,10}$ In corneal ulcers resistant to aforementioned therapies, AMTX may help to prevent or stop corneal melting and eventually even avoid the need for an emergency keratoplasty. ${ }^{11}$

AMTX for ocular surface treatment was first described by de Rötth ${ }^{12}$ and Sorsby. ${ }^{13,14}$ In 1995, Kim and Tseng ${ }^{15}$ developed a method of preserving amniotic membranes using glycerine. Since then, AMTX has been used to treat various ophthalmologic diseases. ${ }^{4,16-19}$

An amniotic membrane consists of a thick basement membrane and an avascular stroma that contains a high concentration of basic fibroblast growth factors. ${ }^{20}$ It also represents a mechanical barrier for frictional forces. ${ }^{21}$ Furthermore, amniotic membranes promote epithelial recovery and suppress inflammation because of the contained cytokines $^{22,23}$ and growth factors. ${ }^{9}$ In addition, they may also have protective antimicrobial properties. ${ }^{21}$ It has been shown that amniotic membranes have antiinflammatory ${ }^{24}$ and antiangiogenic properties. ${ }^{25}$

The aim of this study was to evaluate the outcome of amniotic membrane transplantation in a large cohort of patients with corneal ulcers.

\section{METHODS}

This is a retrospective analysis of consecutive patients who underwent AMTX because of treatment-refractory corneal ulcers at the Department of Ophthalmology, Inselspital Bern University Hospital, University of Bern, Switzerland, between 2012 and 2017. Data collection consisted of findings obtained from the clinical history and ophthalmic examination. All patients underwent a clinical examination before surgery, including the assessment of best corrected visual acuity 
(BCVA) and slit lamp examination. The minimum follow-up period was 6 months. The indication for AMTX was nonhealing corneal ulcers that were considered refractory to medical treatment, defined as not showing any tendency of epithelial healing despite adequate conventional treatment of the ocular surface (eg, artificial eye tears, ointments, and bandage contact lens) and the underlying etiologies (eg, antiherpetic treatment, oculoplastic surgery in case of lagophthalmos or any other eyelid problems, and systemic corticosteroid and/or immunomodulating treatment in rheumatic disease). All consecutive patients undergoing AMTX between 2012 and 2017 and fulfilling these criteria were included in the study. Patients with a loss of follow-up after AMTX were excluded from the study.

The impact of the following parameters on the outcome was evaluated: ulcer etiology and duration between ulcer onset and surgery, ulcer size (measured as a fluorescein staining positive area at the slit lamp in $\mathrm{mm}^{2}$ ), corneal neovascularization, and $\mathrm{BCVA}(\operatorname{LogMAR})$ at baseline (defined as the last visit before AMTX).

Amniotic membranes were obtained from human placentas shortly after elective caesarean delivery and were prepared under sterile conditions. Maternal blood was screened for human immunodeficiency virus types 1 and 2, hepatitis virus types $\mathrm{B}$ and $\mathrm{C}$, and syphilis. After preparation and fixation onto a sterile sponge, amniotic membranes were stored in a $15-\mathrm{mL}$ conservation medium $(50 \%$ glycerol/Dulbecco's Modified Eagle's medium [DMEM] $+0.2 \%$ antibiotic-antimycotic [100X solution containing penicillin, amphotericin B, streptomycin; Thermo Fisher Scientific, Waltham, MA]) at $-70^{\circ} \mathrm{C}$. Surgery was performed mainly under topical and in selected cases using general anesthesia, based on the preference of the patients. During the surgical procedure, an amniotic membrane was placed with the epithelium up within the corneal epithelial defect and fixed using nylon 10-0 interrupted sutures. A second amniotic membrane with the epithelium down was placed overlapping the first layer and over the whole cornea and fixed with a continuous nylon 10-0 suture to the conjunctiva. This technique was previously described in detail. ${ }^{4}$ A therapeutic contact lens was placed over both amniotic membranes at the end of the surgery. Patients were treated with preservative-free topical artificial tears and antibiotic drops. The continuous suture was removed after resorption of the upper amniotic membrane; the single sutures of the deeper amniotic membrane and the contact lens were removed after integration/resorption/loss of the deeper amniotic membrane layer and/or full reepithelialization.

Treatment success was defined as complete corneal reepithelialization within 6 months after the intervention (ie, the absence of any fluorescein staining at the slit lamp examination during the follow-up).

Means and SDs were reported using descriptive statistics. The impact of ulcer etiology and duration between ulcer onset and surgery, ulcer size (area in $\mathrm{mm}^{2}$ ), corneal neovascularization, and BCVA at baseline on epithelial closure (success) were assessed with a logistic regression model with post hoc Wald testing.

A one-way analysis of variance for multiple comparisons was used to compare time with AMTX and epithelial closure times between the groups. A paired Student $t$ test was used to analyze the preoperative and postoperative visual acuity for the whole cohort. For subgroup comparisons of BCVA, multiple $t$ tests with a Holm-Sidak correction were used. Statistical analysis was performed using GraphPad Prism software (Version 8.0.1, San Diego, CA) and IBM SPSS Statistics (Version 25, IBM, Armonk, NY). A $P$-value $<0.05$ was considered as significant.

The retrospective study was approved by the local ethics committee and was performed in accordance with the Declaration of Helsinki.

\section{RESULTS}

A total of 149 eyes of 149 patients undergoing AMTX for a nonhealing corneal ulcer between 2012 and 2017 were included in this study. The mean age at AMTX was 68 years (SD \pm 18 , range $2-95$ years). In most eyes (138 eyes, 93\%), a double-layered AMTX was performed, whereas in 11 eyes (7\%) a multilayer technique was chosen to fill deep-ulcers with the first amnion layers. For the outcome analysis, patients were divided into 8 subgroups according to the etiology of ulcer: group 1: herpetic ulcers; group 2: ulcers associated with rheumatic disease; group 3: ulcers after previous penetrating keratoplasty $(\mathrm{PK})$ or other corneal surgery; group 4: bacterial ulcers; group 5: limbal stem cell insufficiency due to chemical burn or trauma; group 6: ulcers due to bullous keratopathy as a complication after ocular surgery other than PK; group 7: ulcers due to lagophthalmos; and group 8: neurotrophic ulcers (Table 1).

\section{Time to AMTX}

The mean duration from the diagnosis of the corneal ulcer to AMTX was $42 \pm 46$ days. In bacterial ulcers, a mean of 65 days was waited until AMTX, whereas in patients with trauma or chemical burns, AMTX was performed after a mean of only 14 days (Table 1). Time from ulcer diagnosis to AMTX did not reveal any significant difference between the subgroups $(P>0.05)$.

\section{Epithelial Closure After AMTX}

In $70 \%(n=105)$ of eyes, a single AMTX procedure was able to achieve closure of the corneal defect. Among these patients, epithelial closure was observed in $21 \%(\mathrm{n}=$ 32 ) within the first month, in further $40 \%(n=60)$ within 1 to 3 months, and in $9 \%$ of patients $(n=13)$ within 3 to 6 months after AMTX. However, no epithelial closure was achieved in the remaining $30 \%$ of patients $(n=44)$ (Table 2$)$.

Ulcers due to bullous keratopathy, bacterial ulcers, herpetic ulcers, and neurotrophic ulcers had the highest overall closure rates $(79 \%, 80 \%, 85 \%$, and $93 \%$, respectively) and achieved epithelial closure mainly within the first 3 months after surgery. Ulcers in patients with rheumatic disease and with ulcers due to wound-healing problems after corneal surgery revealed the lowest closure rates $(52 \%$ and $57 \%$, respectively). Both, ulcers due to chemical burns or trauma and ulcers due to lagophthalmos, revealed closure rates of $63 \%$. The success rate was significantly better in 
TABLE 1. Etiology of Corneal Ulcers Treated With AMTX $(N=149$ Eyes $)$

\begin{tabular}{llcr}
\hline & N (M/F) & Age at AMTX, yr (mean \pm SD) & Time to AMTX, d (mean \pm SD) \\
\hline Herpetic ulcers & $33(14 / 19)$ & $72 \pm 9$ & $49 \pm 9$ \\
Ulcers associated with rheumatic disease & $23(11 / 12)$ & $72 \pm 9$ & $34 \pm 8$ \\
Previous PK or other corneal surgery & $30(9 / 21)$ & $67 \pm 8$ & $50 \pm 9$ \\
Bacterial ulcers & $10(6 / 4)$ & $62 \pm 8$ & $65 \pm 15$ \\
$\quad$ Ulcers in limbal stem cell insufficiency due to chemical & $16(2 / 14)$ & $53 \pm 8$ & $14 \pm 4$ \\
$\quad$ burn or trauma & $14(6 / 8)$ & $76 \pm 9$ & $31 \pm 8$ \\
$\quad$ Ulcers due to bullous keratopathy, as a complication & & $63 \pm 8$ & $52 \pm 10$ \\
$\quad$ after ocular surgery other than PK & $8(3 / 5)$ & $75 \pm 9$ & $46 \pm 10$ \\
Lagophthalmos & $15(7 / 8)$ & Neurotrophic ulcers &
\end{tabular}

M, male; F, female

herpetic ulcers compared with ulcers associated with rheumatic disease and ulcers after corneal surgery $(P=0.011$ and $P=0.017$, respectively). In addition, neurotrophic ulcers revealed a higher reepithelialization rate than that of ulcers associated with rheumatic disease $(P=0.022)$. No significant difference was observed between all other subgroups.

Time to epithelial closure was significantly slower in ulcers due to bullous keratopathy than that in neurotrophic ulcers $(P=0.01)$, ulcers after corneal surgery $(P=0.007)$, ulcers after chemical burn or trauma $(P=0.006)$, and ulcers associated with rheumatic disease $(P=0.02)$. All other subgroups did not differ significantly between each other in epithelial closure time (Figure 1).

The duration between ulcer onset and surgery, ulcer size (area in $\mathrm{mm}^{2}$, mean $20.3 \mathrm{~mm}^{2}$, and SD $29 \mathrm{~mm}^{2}$ ), presence of corneal neovascularization $(n=95)$, and BCVA at baseline did not reveal to have any significant impact on the

TABLE 2. Epithelial Closure in the Subgroups of Eyes With $\operatorname{AMTX}(\mathrm{N}=149$ Eyes $)$

\begin{tabular}{|c|c|c|c|c|c|}
\hline & \multicolumn{4}{|c|}{ Success } & \multirow{2}{*}{$\begin{array}{c}\text { Failure } \\
\text { Treatment } \\
\text { Failure } \\
(\%)\end{array}$} \\
\hline & $\begin{array}{c}\text { Total } \\
\text { Closure } \\
\text { Rate }(\%)\end{array}$ & $\begin{array}{c}<1 \\
\text { mo } \\
(\%)\end{array}$ & $\begin{array}{c}1-3 \\
\text { mo } \\
(\%)\end{array}$ & $\begin{array}{c}\text { 3-6 } \\
\text { mo } \\
(\%)\end{array}$ & \\
\hline Herpetic ulcers & 84.8 & 30.3 & 39.4 & 15.2 & 15.2 \\
\hline $\begin{array}{l}\text { Ulcers associated with } \\
\text { rheumatic disease }\end{array}$ & 52.2 & 13.0 & 39.1 & 0.0 & 47.8 \\
\hline $\begin{array}{l}\text { Previous PK or other } \\
\text { corneal surgery }\end{array}$ & 56.7 & 16.7 & 40.0 & 0.0 & 43.3 \\
\hline Bacterial ulcers & 80.0 & 20.0 & 50.0 & 10.0 & 20.0 \\
\hline $\begin{array}{l}\text { Ulcers in limbal stem cell } \\
\text { insufficiency due to } \\
\text { chemical burn or } \\
\text { trauma }\end{array}$ & 62.5 & 25.0 & 37.5 & 0.0 & 37.5 \\
\hline $\begin{array}{l}\text { Ulcers due to bullous } \\
\text { keratopathy, as a } \\
\text { complication after } \\
\text { ocular surgery other } \\
\text { than PK }\end{array}$ & 78.6 & 0.0 & 42.9 & 35.7 & 21.4 \\
\hline Lagophthalmos & 62.5 & 12.5 & 37.5 & 12.5 & 37.5 \\
\hline Neurotrophic ulcers & 93.3 & 46.7 & 40.0 & 6.7 & 6.7 \\
\hline Total & 70.5 & 21.5 & 40.3 & 8.7 & 29.5 \\
\hline
\end{tabular}

epithelial closure success rate (ie, AMTX success vs. failure) in our patients $(P>0.05$, each).

\section{Treatment Failure of AMTX}

Because of treatment failure, 42 secondary surgical procedures in 44 eyes with AMTX had to be performed to achieve epithelial closure. A second AMTX was performed whether there was still a realistic chance for epithelial healing. In cases with a high risk of perforation despite the first AMTX or in which perforation already had occurred, PK was performed. If the eye did not have a favorable visual prognosis

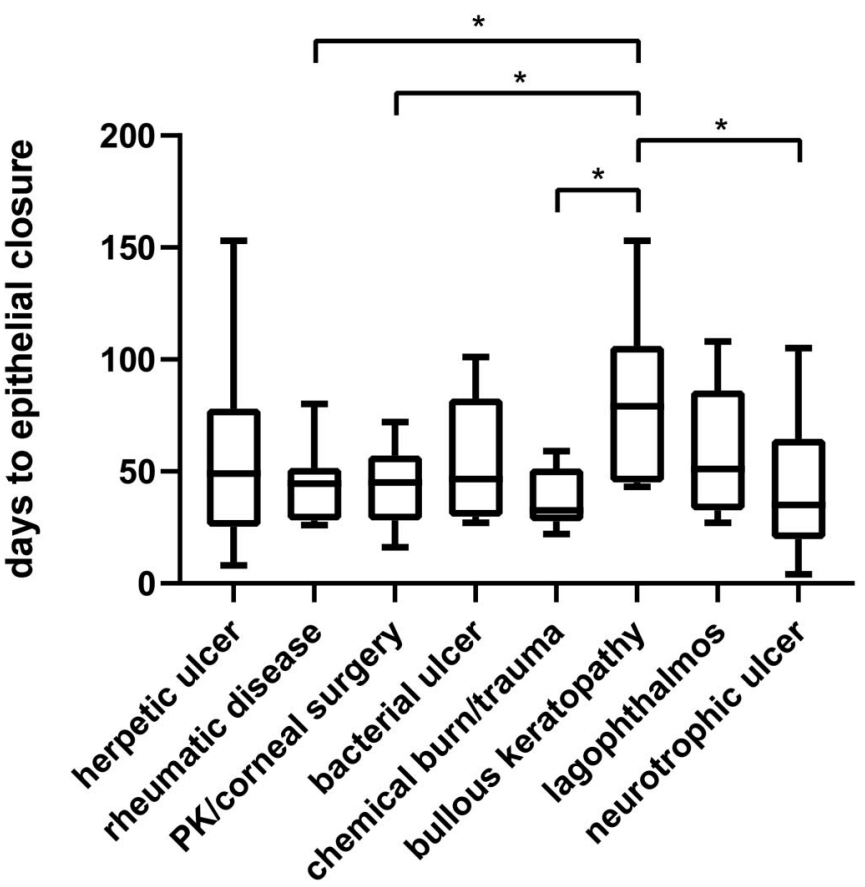

FIGURE 1. Epithelial closure time. Box and whiskers plot for all epithelial closure times in the subgroups. The boxes show 25 and 75 percentiles and the median. The whiskers depict minimum and maximum values. Statistically significant results using the analysis of variance for multiple comparisons are indicated with $*(P<0.05)$. 
and was very painful, conjunctival flap surgery or even evisceration was discussed with the patient. In 2 eyes, epithelial closure was observed 192 and 202 days after the first AMTX (ie, defined as AMTX failure). In 10 eyes, a third procedure was necessary. In only one eye, the third AMTX failed to close the epithelial defect in an ulcer associated with rheumatic disease; therefore, ultimately, PK was performed (Figure 2).

\section{Visual Acuity Outcome}

At baseline, no statistically significant difference in BCVA between the subgroups could be found, except ulcers associated with rheumatic disease with a better BCVA compared with ulcers in bullous keratopathy $(P=0.02)$. When analyzing the BCVA for every subgroup at baseline versus the last follow-up (or at the visit before secondary intervention in cases of AMTX failure with further surgery), no statistically significant change was found $(P>0.05$, each; Figure 3$)$. However, for the whole cohort of patients, BCVA improved significantly from a mean of $1.8 \pm 0.6 \mathrm{LogMAR}$ at baseline to a mean of $1.6 \pm 0.8$ LogMAR (measured at the time-point of the last follow-up or at the last visit before secondary surgical interventions in eyes with AMTX treatment failure undergoing further surgeries) $(P=0.002)$.

\section{DISCUSSION}

Our results reveal an overall AMTX success rate of $70 \%$ in our study population. In most patients, epithelial closure was achieved within the first 3 months after AMTX. Success rates in our patients varied depending on the etiological subgroup.

In nearly $50 \%$ of all patients with ulcers associated with rheumatic disease or due to wound-healing problems after corneal surgery, epithelial closure was not achieved with AMTX. Furthermore, these subgroups revealed a significantly lower epithelial healing rate than those with herpetic ulcers. In ulcers associated with rheumatic disease, this might be because of severe and aggressive corneal inflammation and melting. In ulcers after corneal surgery, epithelial healing is often delayed because of multifactorial etiologies such as

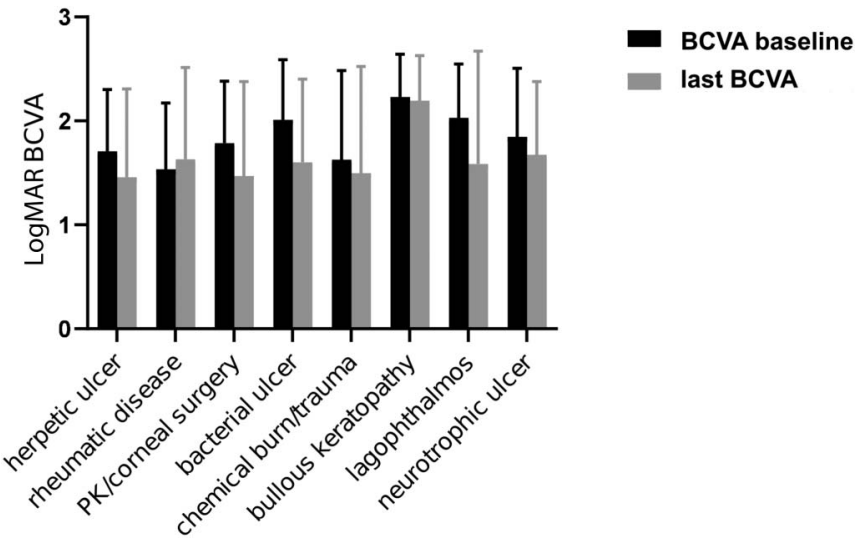

FIGURE 3. Bar chart of BCVA in LogMAR at baseline and at the last follow-up (or at the visit before a secondary intervention in cases of AMTX failure with further surgery). Baseline was defined as BCVA at the last visit before AMTX. No statistically significant difference between the preoperative and postoperative values was found in the subgroups' analyses.

neurotrophic problems and endothelial failure inhibiting corneal reepithelialization.

Most of the previous publications on AMTX are case reports, case series, and reports on AMTX in patients for selected underlying etiologies. ${ }^{4,9-11,16,20,21,26,27}$ In those studies, recurrence rates of $10 \%$ to $29 \%$ after AMTX were reported. ${ }^{4,28}$ These results are slightly lower than our findings that showed an AMTX failure rate of $30 \%$. Moreover, our study showed that there is a significant difference in the success rate between different etiological subgroups, with herpetic ulcers having a significantly higher success rate than ulcers after PK and ulcers associated with rheumatic disease. In addition, neurotrophic ulcers showed a higher success rate after AMTX than ulcers associated with rheumatic disease.

In previous studies, BCVA did not reveal any significant improvement or worsening in the $\mathrm{mid} /$ long term. ${ }^{25,28,29}$ Although AMTX leads to opacification of the corneal surface in the initial phase, the amniotic membrane

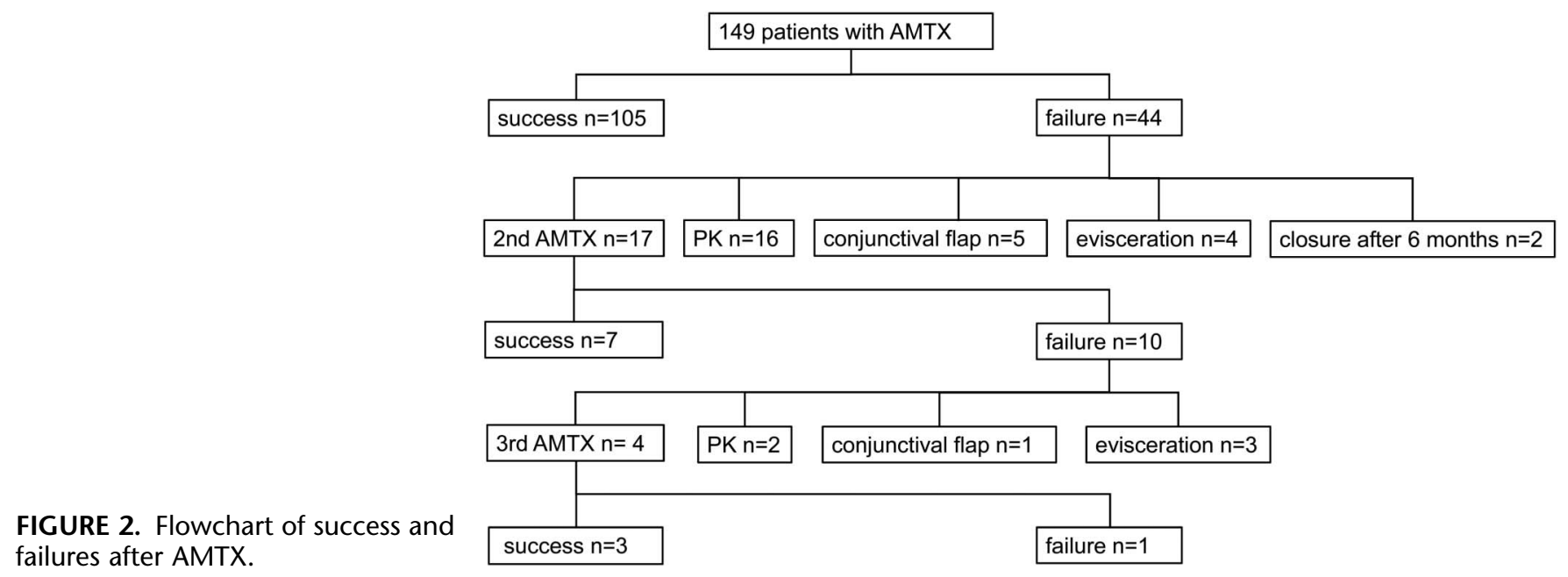


dissolves after a few weeks. Indeed, AMTX may even lead to an improvement of visual acuity through corneal surface restoration. ${ }^{4}$ In addition, in our study, such an improvement was seen, when analyzing the whole cohort of patients. However, this could not be shown in the subgroups' analyses, probably because of the lower power of the smaller sample size.

Our study has limitations because of the retrospective nature of our analysis and the lack of a control group. Another limitation is the lack of optical coherence tomography data to quantify the depth of ulcers. In a clinical slit-lamp examination, descemetocele was observed in only 2 patients before surgery (one patient in the subgroup of ulcers due to bullous keratopathy and one patient within the subgroup of herpetic ulcers). Furthermore, our comparative analytical results should be read with caution, as a potential bias cannot be fully excluded in a noncontrolled retrospective study. On the other hand, our study provides interesting data on a large patient cohort with a variety of different subgroups and information on epithelial closure time.

In conclusion, in this study, we show significant differences in corneal epithelial healing (success rates and time to epithelial closure) after AMTX depending on the underlying etiology, with the best success in patients with neurotrophic ulcers and the worst epithelial closure rates in patients with ulcers associated with rheumatic disease. Future prospective studies with a standardized follow-up are desirable to further investigate epithelial healing dynamics after AMTX.

\section{REFERENCES}

1. Portnoy SL, Insler MS, Kaufman HE. Surgical management of corneal ulceration and perforation. Surv Ophthalmol. 1989;34:47-58.

2. Donzis PB, Mondino BJ. Management of noninfectious corneal ulcers. Surv Ophthalmol. 1987;32:94-110.

3. Kenyon KR. Inflammatory mechanisms in corneal ulceration. Trans Am Ophthalmol Soc. 1985;83:610-663.

4. Lee SH, Tseng SC. Amniotic membrane transplantation for persistent epithelial defects with ulceration. Am J Ophthalmol. 1997;123:303-312.

5. Leibowitz HM, Berrospi AR. Initial treatment of descemetocele with hydrophilic contact lenses. Ann Ophthalmol. 1975;7:1161-1166.

6. Nizeyimana H, Zhou DD, Liu XF, et al. Clinical efficacy of conjunctival flap surgery in the treatment of refractory fungal keratitis. Exp Ther Med. 2017; 14:1109-1113.

7. Pakarinen M, Tervo T, Tarkkanen A. Tarsorraphy in the treatment of persistent corneal lesions. Acta Ophthalmol Suppl. 1987;182:69-73.

8. Knox Cartwright NE, Tole DM, Georgoudis P, et al. Peripheral ulcerative keratitis and corneal melt: a 10-year single center review with historical comparison. Cornea. 2014;33:27-31.

9. Solomon A, Meller D, Prabhasawat P, et al. Amniotic membrane grafts for nontraumatic corneal perforations, descemetoceles, and deep ulcers. Ophthalmology. 2002;109:694-703.

10. Berguiga M, Mameletzi E, Nicolas M, et al. Long-term follow-up of multilayer amniotic membrane transplantation (MLAMT) for non- traumatic corneal perforations or deep ulcers with descemetocele [in German]. Klin Monbl Augenheilkd. 2013;230:413-418.

11. Hoffmann S, Szentmary N, Seitz B. Amniotic membrane transplantation for the treatment of infectious ulcerative keratitis before elective penetrating keratoplasty. Cornea. 2013;32:1321-1325.

12. de Rötth A. Plastic repair of conjunctival defects with fetal membranes Arch Ophthalmol. 1940;23:522-525.

13. Sorsby A, Symons HM. Amniotic membrane grafts in caustic burns of the eye: (burns of the second degree). Br J Ophthalmol. 1946;30:337-345.

14. Sorsby A, Haythorne J, Reed H. Further experience with amniotic membrane grafts in caustic burns of the eye. Br J Ophthalmol. 1947;31: 409-418.

15. Kim JC, Tseng SC. Transplantation of preserved human amniotic membrane for surface reconstruction in severely damaged rabbit corneas. Cornea. 1995; 14:473-484.

16. Kruse FE, Rohrschneider K, Volcker HE. Multilayer amniotic membrane transplantation for reconstruction of deep corneal ulcers. Ophthalmology. 1999;106:1504-1510.

17. Prabhasawat $\mathrm{P}$, Barton $\mathrm{K}$, Burkett $\mathrm{G}$, et al. Comparison of conjunctival autografts, amniotic membrane grafts, and primary closure for pterygium excision. Ophthalmology. 1997;104:974-985.

18. Shimazaki J, Yang HY, Tsubota K. Amniotic membrane transplantation for ocular surface reconstruction in patients with chemical and thermal burns. Ophthalmology. 1997;104:2068-2076.

19. Tseng SC, Prabhasawat P, Lee SH. Amniotic membrane transplantation for conjunctival surface reconstruction. Am J Ophthalmol. 1997;124: $765-774$.

20. Kim JS, Kim JC, Hahn TW, et al. Amniotic membrane transplantation in infectious corneal ulcer. Cornea. 2001;20:720-726.

21. Abdulhalim BE, Wagih MM, Gad AA, et al. Amniotic membrane graft to conjunctival flap in treatment of non-viral resistant infectious keratitis: a randomised clinical study. Br J Ophthalmol. 2015;99:59-63.

22. Solomon A, Rosenblatt M, Monroy D, et al. Suppression of interleukin 1 alpha and interleukin 1beta in human limbal epithelial cells cultured on the amniotic membrane stromal matrix. $\mathrm{Br} J$ Ophthalmol. 2001;85: 444-449.

23. Kim JS, Kim JC, Na BK, et al. Amniotic membrane patching promotes healing and inhibits proteinase activity on wound healing following acute corneal alkali burn. Exp Eye Res. 2000;70:329-337.

24. Ma DH, Wang SF, Su WY, et al. Amniotic membrane graft for the management of scleral melting and corneal perforation in recalcitrant infectious scleral and corneoscleral ulcers. Cornea. 2002;21: 275-283.

25. Uhlig CE, Frings C, Rohloff N, et al. Long-term efficacy of glycerineprocessed amniotic membrane transplantation in patients with corneal ulcer. Acta Ophthalmol. 2015;93:e481-e487.

26. Mohan S, Budhiraja I, Saxena A, et al. Role of multilayered amniotic membrane transplantation for the treatment of resistant corneal ulcers in North India. Int Ophthalmol. 2014;34:485-491.

27. Heinz C, Eckstein A, Steuhl KP, et al. Amniotic membrane transplantation for reconstruction of corneal ulcer in graves ophthalmopathy. Cornea. 2004;23:524-526.

28. Letko E, Stechschulte SU, Kenyon KR, et al. Amniotic membrane inlay and overlay grafting for corneal epithelial defects and stromal ulcers. Arch Ophthalmol. 2001;119:659-663.

29. Prabhasawat P, Tesavibul N, Komolsuradej W. Single and multilayer amniotic membrane transplantation for persistent corneal epithelial defect with and without stromal thinning and perforation. $\mathrm{Br} J$ Ophthalmol. 2001;85:1455-1463. 